\title{
The macroeconomic impact of the green building industry
}

$$
\text { Song } q i^{1, a} \text {, Han yuan yuan }{ }^{2, b}
$$

${ }^{1}$ Xi'an University of Architecture and Technology, Xi'an 710055, China;

${ }^{2}$ School of management, Xi'an University of Architecture and Technology, Xi'an 710055, China.

$$
\text { a } 413531307 @ q q . c o m,{ }^{b} 598160057 @ q q . c o m
$$

Keywords: Green Building, Policy Analysis, Macroeconomic Analysis, Low-carbon city.

\begin{abstract}
In recent years, green building flourish in our country, but mainly in the technical aspects of the application and cost analysis, and for estimates of the macroeconomic impact of green buildings rarely, paper for qualitative analysis of this problem, use 2010 input-output table data on green buildings is estimated to give some policy recommendations based on measurement results.
\end{abstract}

\section{Introduction}

With the rapid development of China's economy and the acceleration of urbanization, Human activities on the earth's resources cause excessive consumption of global warming. Among the energy consumption, Transportation and industrial buildings are called the three "big consume". Therefore, the development of low-energy buildings for energy conservation plays a vital role.But now most of the green building study focused on technology applications and environmental benefits, The macro-benefit study on green building industry rarely,This paper uses 2010 input-output table values to measure the driving effect of the green building industry to other industries ${ }^{[1]}$.

\section{Driving effect on the macroeconomy green building industry}

\subsection{After the driving effect.}

After the driving effect is through direct consumption relationships and to measure the relationship completely consumed. Direct consumption coefficient calculation formula[2]:

$$
a_{i j}=\frac{X_{i j}}{X_{j}}(i, j=1,2, \ldots . ., n)
$$

Complete consumption coefficient calculation formula:

$$
B=(I-A)^{-1}-I
$$

\subsection{Forward driving effect.}

Forward driving effect refers to the direct distribution relationships and to measure the relationship between the fully allocated.Direct allocation formula coefficient:

$$
r_{i j}=\frac{X_{i j}}{X_{i}}(i, j=1,2, \ldots \ldots, n)
$$

Complete partition coefficient is calculated:

$$
D=(I-R)^{-1}-I
$$

\subsection{Total driving effect.}

Total driving effect is the sum of the driving effect and forward drive after effects.

\section{Empirical Analysis}

\subsection{Direct consumption Relations}

In 2010 Chinese input-output table for the analysis of the object, The same calculation method using the direct consumption coefficient Direct consumption coefficient can be calculated with the real estate industry sectors.[3] 


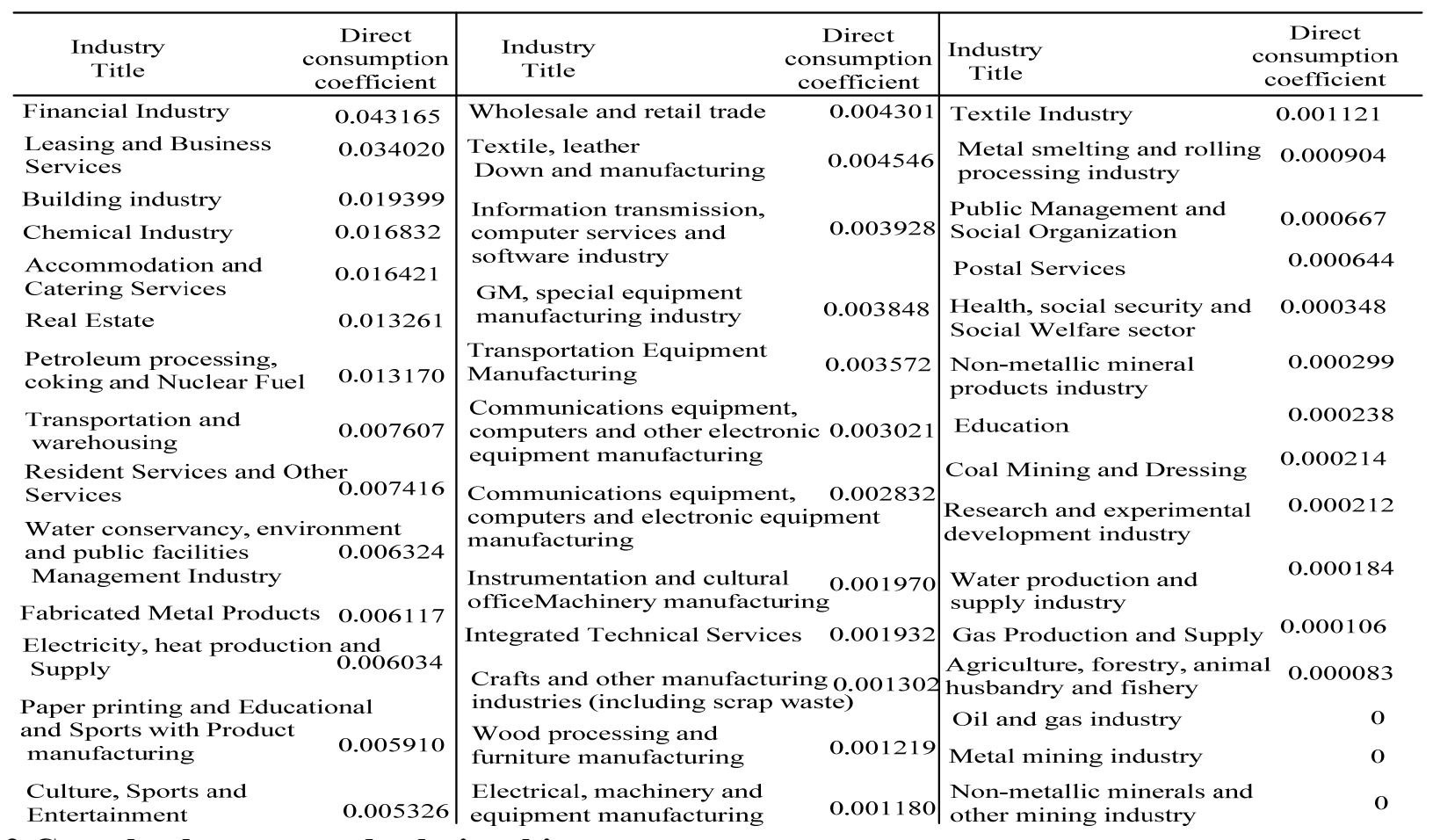

\subsection{Completely consumed relationship}

Similarly, in the analysis of the four sectors of the total consumption coefficient,We are 41 departments to do further analysis.In 2010 Chinese input-output table (41 sectors) for the analysis of the object,Calculated using complete consumption coefficient.

\begin{tabular}{|c|c|c|c|c|c|}
\hline $\begin{array}{l}\text { Industry } \\
\text { Title }\end{array}$ & $\begin{array}{l}\text { Total } \\
\text { onsumption } \\
\text { coefficient }\end{array}$ & $\begin{array}{l}\text { Industry } \\
\text { Title }\end{array}$ & $\begin{array}{c}\text { Total } \\
\text { consumption } \\
\text { coefficient }\end{array}$ & $\begin{array}{l}\text { Industry } \\
\text { Title }\end{array}$ & $\begin{array}{l}\text { Total } \\
\text { nsumption } \\
\text { oefficient }\end{array}$ \\
\hline Chemical Industry & 0.078152 & \multirow{2}{*}{\multicolumn{2}{|c|}{$\begin{array}{l}\text { Communications equipment, } \\
\text { computers and other electronic } 0.021882 \\
\text { equipment manufacturing }\end{array}$}} & $\begin{array}{l}\text { Culture, Sports and } \\
\text { Entertainment }\end{array}$ & 0.007161 \\
\hline $\begin{array}{l}\text { Agriculture, forestry, animal } \\
\text { husbandry and fishery }\end{array}$ & 0.073324 & & & \multirow{2}{*}{\multicolumn{2}{|c|}{$\begin{array}{l}\text { Instrumentation and cultural office } \\
\text { machinery manufacturing } \quad 0.006182\end{array}$}} \\
\hline Financial Industry & & Building industry & 0.020878 & & \\
\hline I osino and Rucinoss Sorvisos & 0.062683 & Real Estate & 0.018806 & \multirow{2}{*}{\multicolumn{2}{|c|}{$\begin{array}{l}\text { Wood processing and } \\
\text { furniture manufacturing } \quad 0.005908\end{array}$}} \\
\hline Leasing and Business Services & 0.047163 & Wholesale and retail trade & 0.017625 & & \\
\hline $\begin{array}{l}\text { Metal smelting and rolling } \\
\text { processing industry }\end{array}$ & 0.039592 & Fabricated Metal Products & 0.017588 & \multicolumn{2}{|c|}{ Integrated Technical Services 0.005770} \\
\hline $\begin{array}{l}\text { Electricity, heat production and } \\
\text { Supply }\end{array}$ & \multirow[t]{2}{*}{0.036484} & $\begin{array}{l}\text { Transportation Equipment } \\
\text { Manufacturing }\end{array}$ & 0.017565 & $\begin{array}{l}\text { Non-metallic Minerals and } \\
\text { Other Ore Mining industry }\end{array}$ & 0.002655 \\
\hline Petroleum processing, coking & & Coal Mining and Dressing & 0.015961 & Postal Services & 0.001563 \\
\hline $\begin{array}{l}\text { and nuclear fuel Material } \\
\text { processing industry }\end{array}$ & 0.035154 & $\begin{array}{l}\text { Non-metallic mineral } \\
\text { products industry }\end{array}$ & 0.012133 & $\begin{array}{l}\text { Research and experimental } \\
\text { development industry }\end{array}$ & 0.001425 \\
\hline Transportation and warehousin & $\lg _{0.031955}$ & Resident Services and & 0.011617 & \multirow{2}{*}{\multicolumn{2}{|c|}{$\begin{array}{l}\text { Water conservancy, environment } \\
\text { and public facilities } \\
\text { Management Industry }\end{array}$}} \\
\hline $\begin{array}{l}\text { Food manufacturing and } \\
\text { tobacco processing industry }\end{array}$ & 0.031112 & $\begin{array}{l}\text { Other Services } \\
\text { Textile Industry }\end{array}$ & 0.011539 & & \\
\hline Accommodation and & ( & Metal mining industry & 0.00945 & Gas Production and Supply & 0.001022 \\
\hline Flectrical machinerv and & 0.021220 & $\begin{array}{l}\text { Textile, leather } \\
\text { Down and manufacturing }\end{array}$ & 0.009325 & \multicolumn{2}{|c|}{$\begin{array}{l}\text { Health, social security and social } \\
\text { Welfare sector }\end{array}$} \\
\hline $\begin{array}{l}\text { Electrical, machinery and } \\
\text { equipment manufacturing }\end{array}$ & 0.025437 & \multirow{2}{*}{\multicolumn{2}{|c|}{$\begin{array}{l}\text { Information transmission, } \\
\text { computer services and }\end{array}$}} & \multirow{2}{*}{\multicolumn{2}{|c|}{$\begin{array}{l}\text { Public Management and } \\
\text { Social Organization }\end{array}$}} \\
\hline $\begin{array}{l}\text { GM, special equipment } \\
\text { manufacturing industry }\end{array}$ & 0.024015 & & & & \\
\hline $\begin{array}{l}\text { Paper printing and Educational } \\
\text { Sports manufacturing }\end{array}$ & al and 0.023533 & \multirow{2}{*}{$\begin{array}{l}\text { Crafts and other } \\
\text { manufacturing industries } \\
\text { (including scrap waste) }\end{array}$} & \multirow[b]{2}{*}{0.007900} & $\begin{array}{l}\text { Water production and } \\
\text { supply industry }\end{array}$ & 0.000749 \\
\hline Oil and gas industry & 0.022384 & & & Education & 0.000574 \\
\hline
\end{tabular}

\section{3 direct distribution relationship}

In 2010 Chinese input-output tables for the analysis of the object,Using the same calculation method of direct distribution coefficients, Direct distribution coefficient can be calculated with the real estate industry sectors. 


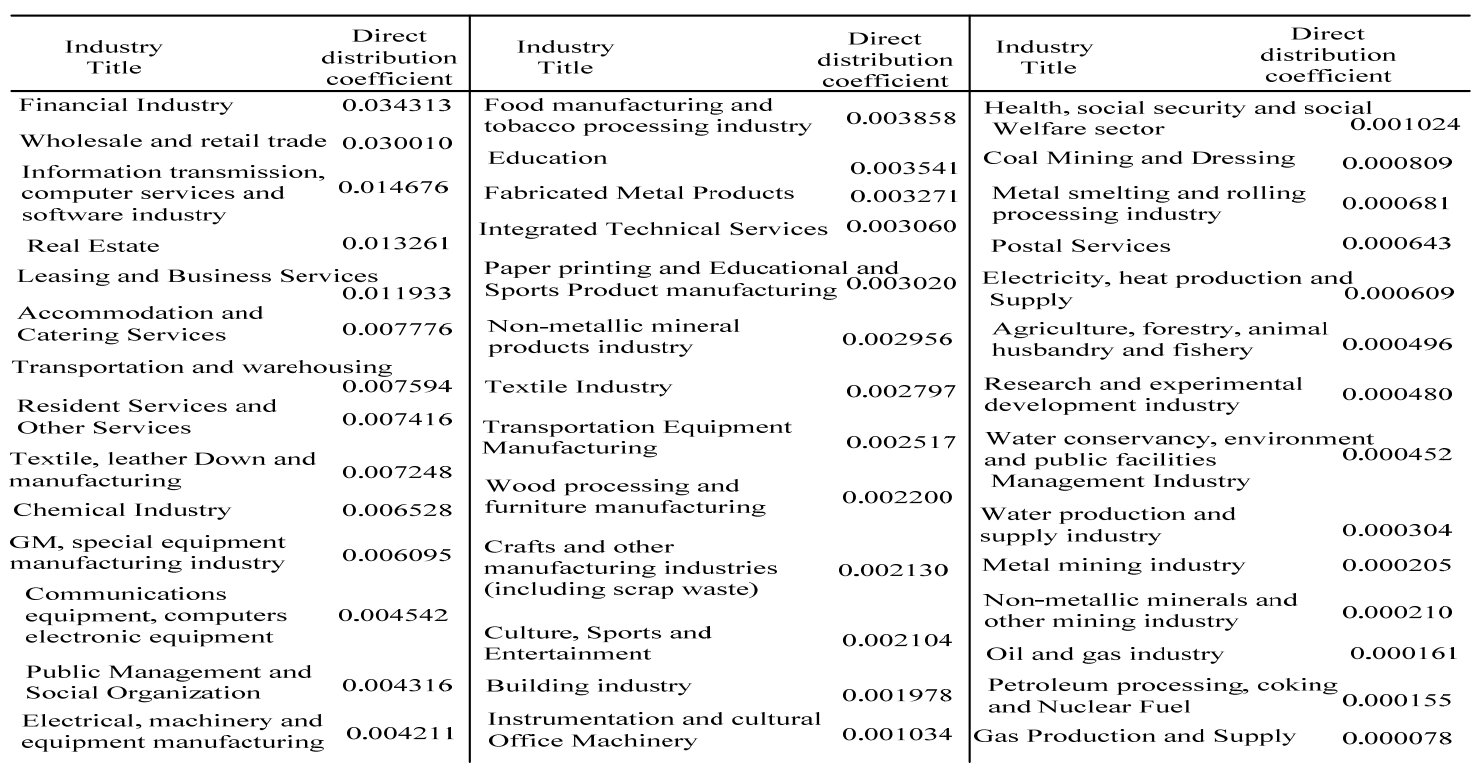

\section{4 fully allocated relationship}

In 2010 Chinese input-output tables for the analysis of the object, The same calculation method using complete partition coefficient, You can calculate the exact distribution coefficient of real estate and various industrial sectors.

\begin{tabular}{|c|c|c|c|c|}
\hline $\begin{array}{l}\text { Industry } \\
\text { Title }\end{array}$ & $\begin{array}{l}\text { Complete } \\
\text { partition } \\
\text { coefficient }\end{array}$ & $\begin{array}{l}\text { Industry } \\
\text { Title }\end{array}$ & $\begin{array}{l}\text { Complete } \\
\text { partition } \\
\text { coefficient }\end{array}$ & $\begin{array}{l}\text { Complete } \\
\text { partition } \\
\text { coefficient }\end{array}$ \\
\hline Leasing and Business Services & O. 064414 & Fabricated Metal Products & 0.020898 & \multirow{2}{*}{$\begin{array}{l}\text { Communications equipment, compu- } \\
\text { ters and electronic equipment } \\
0.011408\end{array}$} \\
\hline Financial Industry & 0.057406 & \multicolumn{2}{|c|}{ Transportation and warehousing 0.018758} & \\
\hline Oil and gas industry & 0.055552 & Real Estate & 0.018625 & $\begin{array}{l}\text { Wood processing and } \\
\text { furniture manufacturing } \quad 0.011405\end{array}$ \\
\hline Culture, Sports and Entertainment & 0.043258 & $\begin{array}{l}\text { Integrated Technical Services } \\
\text { Crafts and other }\end{array}$ & 0.017510 & $\begin{array}{l}\text { Food manufacturing and } \\
\text { tobacco processing industry } 0.010817\end{array}$ \\
\hline & 0.037146 & $\begin{array}{l}\text { manufacturing industries } \\
\text { (including scrap waste) }\end{array}$ & 0.016720 & \multirow{2}{*}{$\begin{array}{l}\text { Agriculture, forestry, animal } \\
\text { husbandry and fishery } 0.010443\end{array}$} \\
\hline Postal Services & 0.036052 & Electrical, machinery an & & \\
\hline $\begin{array}{l}\text { Petroleum processing, co } \\
\text { and nuclear fuel Material }\end{array}$ & 0.034017 & equipment manufacturing & 0.016364 & $\begin{array}{l}\text { GM, special equipment } \\
\text { manufacturing industry }\end{array}$ \\
\hline & & & & Textile Industry $\quad 0.010367$ \\
\hline $\begin{array}{l}\text { Paper printing and Educational anc } \\
\text { Sports manufacturing }\end{array}$ & d 0.033127 & $\begin{array}{l}\text { industry } \\
\text { Metal smelting } \\
\text { processing ind }\end{array}$ & 0.014045 & $\begin{array}{l}\text { Water conservancy, environment } \\
\text { and public facilities Management } \\
\text { Industry }\end{array}$ \\
\hline $\begin{array}{l}\text { Resident Services and Other } \\
\text { Services }\end{array}$ & 0.028586 & $\begin{array}{l}\text { Non-metallic } N \\
\text { Other Ore Min }\end{array}$ & 0.013511 & $\begin{array}{l}\text { Non-metallic mineral products } \\
\text { industry } \\
0.008815\end{array}$ \\
\hline $\begin{array}{l}\text { Instrument } \\
\text { office mac }\end{array}$ & 0.025279 & $\begin{array}{l}\text { Rese } \\
\text { deve }\end{array}$ & 0.012470 & $\begin{array}{l}\text { Transportation Equipment } \\
\text { Manufacturing }\end{array}$ \\
\hline Metal mining industry & 0.024033 & $\begin{array}{l}\text { Water production and supply } \\
\text { industry }\end{array}$ & 0.01238 & Building industry $\quad 0.006128$ \\
\hline $\begin{array}{l}\text { Electricity, heat production and } \\
\text { Supply }\end{array}$ & 0.023653 & Gas Production and Supply & 0.012257 & $\begin{array}{l}\text { Health, social security and social } \\
\text { Welfare sector }\end{array}$ \\
\hline Chemical Industry & 0.022631 & Wholesale and retail trade & 0.011429 & $\begin{array}{l}\text { Education } \\
\text { Public Manageme }\end{array}$ \\
\hline Coal Mining and Dressing & 0.022544 & & 0.011409 & Social Organization \\
\hline
\end{tabular}


3.5 Total driving effect:The sum of After and forward driving effect

\begin{tabular}{|c|c|c|c|c|c|}
\hline $\begin{array}{l}\text { Industry } \\
\text { Title }\end{array}$ & $\begin{array}{c}\text { Total } \\
\text { nsumption } \\
\text { oefficient }\end{array}$ & $\begin{array}{l}\text { Industry } \\
\text { Title }\end{array}$ & $\begin{array}{c}\text { Total } \\
\text { consumption } \\
\text { coefficient }\end{array}$ & $\begin{array}{l}\text { Industry } \\
\text { Title }\end{array}$ & $\begin{array}{l}\text { Total } \\
\text { nsumption } \\
\text { oefficient }\end{array}$ \\
\hline & 0.078152 & \multirow{2}{*}{\multicolumn{2}{|c|}{$\begin{array}{l}\text { Communications equipment, } \\
\text { computers and other electronic } 0.021882 \\
\text { equipment manufacturing }\end{array}$}} & $\begin{array}{l}\text { Culture, Sports and } \\
\text { Entertainment }\end{array}$ & \\
\hline $\begin{array}{l}\text { Agriculture, forestry, animal } \\
\text { husbandry and fishery }\end{array}$ & 0.073324 & & & \multirow{2}{*}{\multicolumn{2}{|c|}{$\begin{array}{l}\text { Instrumentation and cultural office } \\
\text { machinery manufacturing } \quad 0.006182\end{array}$}} \\
\hline Financial Industry & 0.062683 & $\begin{array}{l}\text { Building industry } \\
\text { Real Estate }\end{array}$ & $\begin{array}{c}0.020878 \\
0.018806\end{array}$ & & \\
\hline Leasing and Business Services & 0.047163 & Wholesale and retail trade & 0.017625 & \multicolumn{2}{|c|}{$\begin{array}{l}\text { Wood processing and } \\
\text { furniture manufacturing }\end{array} \quad 0.005908$} \\
\hline $\begin{array}{l}\text { Metal smelting and rolling } \\
\text { processing industry }\end{array}$ & 0.039592 & Fabricated Metal Products & 0.017588 & \multicolumn{2}{|c|}{ Integrated Technical Services 0.005770} \\
\hline $\begin{array}{l}\text { Electricity, heat production and } \\
\text { Supply }\end{array}$ & 0.036484 & $\begin{array}{l}\text { Transportation Equipment } \\
\text { Manufacturing }\end{array}$ & 0.017565 & $\begin{array}{l}\text { Non-metallic Minerals and } \\
\text { Other Ore Mining industry }\end{array}$ & 0.002655 \\
\hline Petroleum processing, coking & & Coal Mining and Dressing & 0.015961 & Postal Services & 0.001563 \\
\hline $\begin{array}{l}\text { and nuclear fuel Material } \\
\text { processing industry }\end{array}$ & 0.035154 & $\begin{array}{l}\text { Non-metallic mineral } \\
\text { products industry }\end{array}$ & 0.012133 & $\begin{array}{l}\text { Research and experimental } \\
\text { development industry }\end{array}$ & 0.001425 \\
\hline Transportation and warehousing & $g_{0.031955}$ & $\begin{array}{l}\text { Resident Services and } \\
\text { Other Services }\end{array}$ & 0.011617 & \multirow{2}{*}{\multicolumn{2}{|c|}{$\begin{array}{l}\text { Water conservancy, environment } \\
\text { and public facilities } \\
\text { Management Industry }\end{array}$}} \\
\hline $\begin{array}{l}\text { Food manufacturing and } \\
\text { tobacco processing industry }\end{array}$ & 0.031112 & Textile Industry & 0.011539 & & \\
\hline $\begin{array}{l}\text { Accommodation and } \\
\text { Catering Services }\end{array}$ & 0.027220 & $\begin{array}{l}\text { Metal mining industry } \\
\text { Textile, leather }\end{array}$ & $0.00945 \$$ & $\begin{array}{l}\text { Gas Production and Supply } \\
\text { Health social security and so }\end{array}$ & 0.001022 \\
\hline $\begin{array}{l}\text { Electrical, machinery and } \\
\text { equipment manufacturing }\end{array}$ & 0.025437 & $\begin{array}{l}\text { Down and manufacturing } \\
\text { Information transmission, }\end{array}$ & & \multicolumn{2}{|c|}{$\begin{array}{l}\text { Health, social security and social } \\
\text { Welfare sector }\end{array}$} \\
\hline $\begin{array}{l}\text { GM, special equipment } \\
\text { manufacturing industry }\end{array}$ & 0.024015 & $\begin{array}{l}\text { computer services and } \\
\text { software industry }\end{array}$ & 0.009241 & Social Organization & 0.000835 \\
\hline $\begin{array}{l}\text { Paper printing and Educational } \\
\text { Sports manufacturing }\end{array}$ & and 23533 & $\begin{array}{l}\text { Crafts and other } \\
\text { manufacturing industries }\end{array}$ & & $\begin{array}{l}\text { Water production and } \\
\text { supply industry }\end{array}$ & 0.000749 \\
\hline Oil and gas industry & 0.022384 & (including scrap waste) & 0.007900 & Education & 0.000574 \\
\hline
\end{tabular}

Calculation 2010 input-output table based on results, Green building in China's real estate industry for each additional unit of output on a total driving effect is 1.616712 for each industry.That green building construction in order to improve energy efficiency and reduce emissions into the incremental cost per 1 million, will be the economic value of the various industrial sectors in the entire economy.

\section{Suggestions}

\subsection{Increase government investment.}

Governments should actively promote and advocate in the country's implementation of green building, green building industry financial subsidies.For the application of green building materials, the government should support the development of new energy-saving building materials, technology, technology[4].

\subsection{Establish a sound legal system.}

Although China has issued a corresponding policy of green building, but still far from perfect,To make green building a virtuous circle in the direction of healthy development, it is necessary to improve the legal system,And to combine my aunt actual situation gradually improved.

\subsection{Strengthen publicity and education.}

In China,most people think of green building just unrealistic slogans, Not really implemented.To change this situation, we must strengthen publicity and education, so that people pay attention to green building for a major role in energy conservation will be incorporated into the work of the center of gravity.

\section{References}

[1]. Ye zuda. Macroeconomic of green building research, J. Energy conservation. 2012(10) 97-103.

[2]. Wang meimei: Chinese real estate industry input-output analysis.Chongqing university,China 2009 1-63.

[3]. Liu shuixing. Chinese real estate industry and the national economy other,J.Journal of Shanghai Economic Management College.2003(11)58-63.

[4]. Chen minjie. Economic analysis of low-carbon green buildings,J. Energy and Environment. 2012(02) 116-117. 